\title{
A Theory of Volumetric Growth for Compressible Elastic Biological Materials
}

\author{
STEPHEN M. KLISCH \\ Mechanical Engineering Department, California Polytechnic State University, San Luis Obispo, \\ CA 93407, USA \\ TiMOThY J. VAN DYKE \\ ANNE HoGER \\ Department of Mechanical and Aerospace Engineering, University of California at San Diego, \\ La Jolla, CA 92093-0411, USA
}

\begin{abstract}
A general theory of volumetric growth for compressible elastic materials is presented. The authors derive a complete set of governing equations in the present configuration for an elastic material undergoing a continuous growth process. In particular, they obtain two constitutive restrictions from a work-energy principle. First, the authors show that a growing elastic material behaves as a Green-elastic material. Second, they obtain an expression that relates the stress power due to growth to the rate of energy change due to growth. Then, the governing equations for a small increment of growth are derived from the more general theory. The equations for the incremental growth boundary-value problem provide an intuitive description of the quantities that describe growth and are used to implement the theory. The main features of the theory are illustrated with specific examples employing two strain energy functions that have been used to model biological materials.
\end{abstract}

Key Words: Growth, elasticity, biomechanics

\section{1.- INTRODUCTION-}

Cartilaginous tissues, such as articular cartilage and the intervertebral disk, are composed primarily of three constituents: water, collagen, and proteoglycans. The latter two constituents form a collagen-proteoglycan solid matrix that also contains a low density of cartilage cells called chondrocytes. The results of numerous studies suggest that mechanical loading affects the biosynthetic activity of the chondrocytes and, consequently, the growth and remodeling of the solid matrix. For example, the onset of osteoarthritis is seen at an early age in developing humans with dysplastic hips if the condition is left untreated (Pauwels [1]). Another example is that injury to the growth plate cartilage of developing bones may be accompanied by premature growth arrest and lower limb length discrepancies (Ogden [2]). In the intervertebral disk, the annular biochemistry differs in the concave and convex sides of scoliotic spines, possibly due to different states of mechanical loading (Brickley-Parson 
and Glimcher [3]). Such studies suggest that abnormal mechanical loading conditions have an adverse effect on the growth and remodeling of cartilaginous tissues in vivo. In addition, in vivo models of osteoarthritis (Sandy et al. [4]; Kiviranta et al. [5]), in vivo models of joint immobilization (Kiviranta et al. [6]; Palmoski et al. [7]), and in vitro experiments with tissue and cell cultures (Hall et al. [8]; Sah et al. [9]) provide experimental evidence that growthrelated parameters such as biochemical composition, mechanical properties, and cartilage thickness vary due to changes in the stress, strain, and water content.

Models of the finite deformation and flow-dependent mechanical properties of both articular cartilage (Ateshian [10]) and the intervertebral disk (Klisch and Lotz [11]) have been developed using continuum mixture theory. The collagen-proteoglycan matrix is modeled as a compressible finitely elastic solid and the water as an inviscid fluid. However, no continuum mixture theory has been developed to model the observed changes of tissue during growth and remodeling. As a first step toward developing such a model, a theory of growth for compressible elastic materials is developed in this paper.

Hsu [12] presented the first model of uniform volumetric stress-dependent growth of a linearly elastic material. Cowin and Hegedus [13] developed a bone remodeling theory using a mixture approach; implicit in this work is the assumption that the growth is isotropic and can be represented by the change of a scalar parameter (the volume fraction). Skalak and colleagues [14-16] made several important contributions in the area of growth mechanics; in particular, Skalak et al. [15] observed that growth can lead to the development of residual stresses in the tissue. A general theory of growth for soft tissues was introduced by Rodriguez et al. [17]. In that theory, growth is defined as the addition or removal of material that is mechanically identical to the original material. The kinematics of material deposition is described by a tensor, so anisotropic growth may be modeled. Taber and colleagues have used models based on that theory to study the stress-modulated growth of both the heart (Lin and Taber [18]) and the aorta (Taber and Eggers [19]; Taber [20]) using several growth laws. The theory, as presented in [17] and implemented in [18-20], defined the growth law relative to a fixed configuration of the material. Consequently, the compounding effect of the growth of new material was not accounted for during the growth process. The theory of [17] was further developed by Chen and Hoger [21]. Recently, Hoger [22] further extended the theory and presented an implementation in which the growth law is defined on the current configuration of the growing material; this implementation was used to model the growth of aortic tissue by Van Dyke and Hoger [23]. An underlying assumption appearing in [17-20, 22] was that the growing elastic material is incompressible.

The primary aim of this paper is to develop a theory of growth for compressible elastic materials. The theory may be useful in describing the growth of tissue-engineered constructs and tissue explants obtained from the body under various loading conditions. The governing equations for the theory are developed as a function of position on the current configuration of a growing elastic material. In practice, experiments must be performed at different stages of the growth process to quantify the evolution of the tissue's composition, geometry, and material behavior relative to a predetermined reference configuration. Therefore, we introduce a fixed reference configuration that can be identified with an experimentally characterized configuration of the material and can be used as a reference configuration for the growth boundary-value problem.

Several of the variables that are introduced relative to this fixed reference configuration lack a clear physical interpretation. However, when interpreted in terms of the continuously 
changing current configuration of a material during a growth process, these quantities have clear physical meanings. Thus, a secondary aim of this paper is to obtain the governing equations for a small increment of growth and to provide an intuitive description of the quantities that describe growth. In addition, the governing equations for the incremental problem can be easily used to implement the theory. The kinematics of growth for compressible elastic materials is outlined in Section 2. The balance laws and the formulation of a growth continuity equation are presented in Section 3. Constitutive restrictions are derived from a work-energy principle in Section 4 . The governing equations for an increment of growth are obtained in Section 5. The boundary-value problem of spherically symmetric growth of a spherical shell in the absence of external loading is outlined in Section 6. The solutions of this problem for two different materials are presented and discussed in Section 7. Concluding remarks appear in Section 8.

\section{2.- KINEMATICS-}

In this section, we present the kinematics necessary to describe growth of compressible elastic materials. An important feature of the theory as proposed is that a complete set of governing equations is developed for the present configuration of an elastic material that is undergoing a continuous growth process. The structure of the theory is motivated by how it may be applied in practice. Consider a growing elastic body $\mathcal{B}$ that occupies the configuration $\kappa_{0}(\mathcal{B})$ at time $t_{0}$ (Figure 1). This configuration represents a loaded configuration of the tissue that, when unloaded, occupies the configuration $\kappa_{1}(\mathcal{B})$, in which the geometric and material properties have been experimentally characterized. Suppose that the material in the configuration $\kappa(\mathcal{B})$ at time $t$, achieved after some period of growth, could also be characterized in its unloaded configuration $\kappa_{g}(\mathcal{B})$ (Figure 1). In particular, experiments may be used to determine the changes in tissue mass, geometry, and mechanical properties due to the growth process. We will introduce an arbitrary, fixed reference configuration $\kappa_{R}(\mathcal{B})$ that may represent one of these experimentally determined configurations. In practice, $\kappa_{R}(\mathcal{B})$ may be chosen to coincide with either of the configurations $\kappa_{0}(\mathcal{B})$ or $\kappa_{1}(\mathcal{B})$ of Figure 1.

Motivated by the above remarks, we consider a growing elastic body $\mathcal{B}$, which occupies an arbitrary reference configuration $\kappa_{R}(\mathcal{B})$ with residual stress field $\stackrel{\circ}{\mathbf{T}}$ (Figure 2). The body $\mathcal{B}$ occupies the loaded grown configuration $\kappa(\mathcal{B})$ at a time $t$. A material particle of $\mathcal{B}$ occupies position $\mathbf{X}$-in $\kappa_{R}(\mathcal{B})$ and position $\mathbf{x}$-in $\kappa(\mathcal{B})$. It is assumed that the set of material points in $\kappa_{R}(\mathcal{B})$ is dense in the set of all points in $\kappa(\mathcal{B})$. The motion of $\mathcal{B}$ is defined by a sufficiently smooth, invertible mapping

$$
\mathbf{x}=\chi(\mathbf{X}, t)
$$

with deformation gradient

$$
\mathbf{F}=\frac{\partial \chi(\mathbf{X}, t)}{\partial \mathbf{X}}
$$

that satisfies 


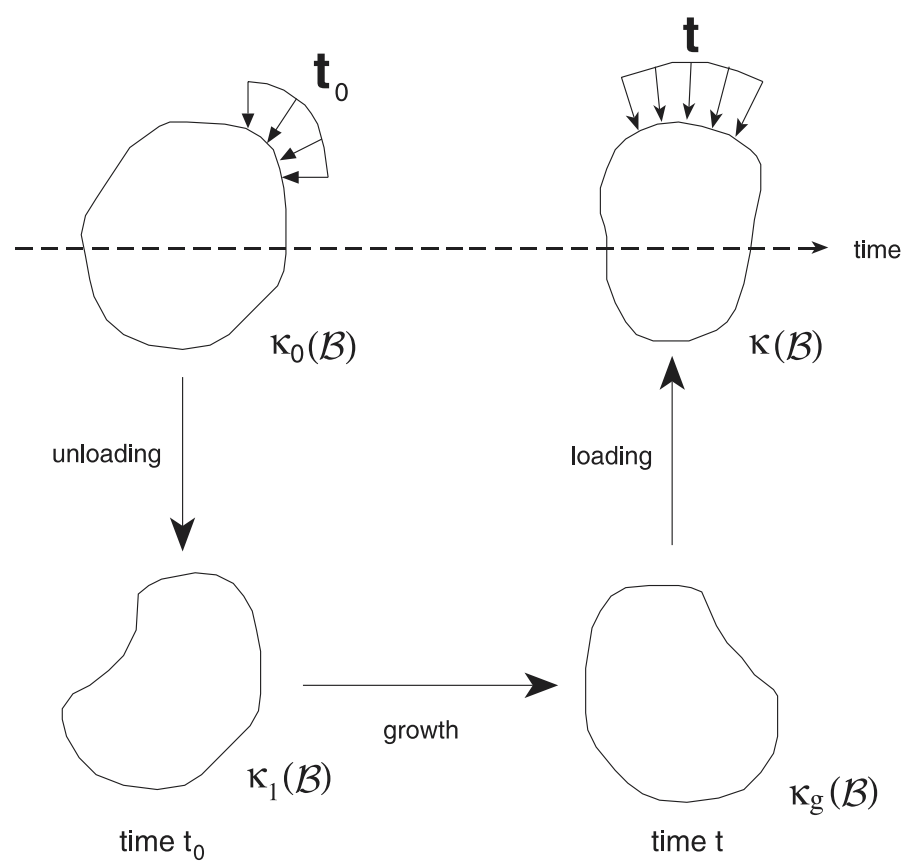

Fig. 1. Schematic of a growing elastic body $\mathcal{B}$ that occupies the configurations $\kappa_{0}(\mathcal{B})$ and $\kappa(\mathcal{B})$ at different times during a continuous growth process. The reference configuration $\kappa_{1}(\mathcal{B})$ is obtained by unloading from $\kappa_{0}(\mathcal{B})$, and the unloaded grown configuration $\kappa_{g}(\mathcal{B})$ is obtained by unloading from $\kappa(\mathcal{B})$.

$$
\mathbf{J}=\operatorname{det} \mathbf{F}>0 .
$$

Given any point of the growing elastic material that occupies the position $\mathbf{x}$-at time $t$, the mapping (2.1) may be inverted to obtain

$$
\mathbf{X}=\chi^{-1}(\mathbf{x}, t)
$$

Thus, functions that are defined on the loaded grown configuration $\kappa(\mathcal{B})$ may be redefined on the reference configuration $\kappa_{R}(\mathcal{B})$ using (2.4). Also, any material point that is added during the growth process is associated with a unique material point in $\kappa_{R}(\mathcal{B})$ through the inverse mapping (2.4).

The deformation gradient $\mathbf{F}$-of the mapping from $\kappa_{R}(\mathcal{B})$ to $\kappa(\mathcal{B})$ is assumed to obey the following decomposition (Figure 2):

$$
\mathbf{F}=\mathbf{F}^{l} \mathbf{M}^{e} \mathbf{M}^{g} .
$$

The tensor $\mathbf{M}^{e} \mathbf{M}^{g}$ describes the total deformation due to growth, relative to $\kappa_{R}(\mathcal{B})$, whereas the deformation gradient $\mathbf{F}^{l}$ represents a superposed elastic deformation produced by applied loads. We assume that this decomposition is such that the amount and orientation of mass 


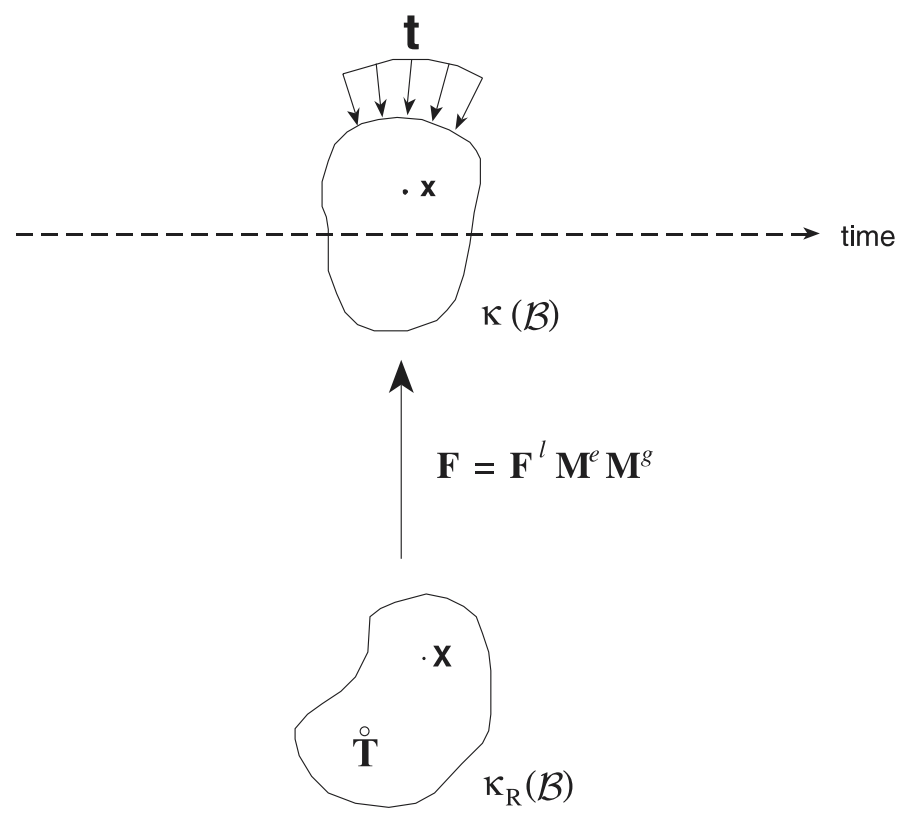

Fig. 2. Schematic of the decomposition of the deformation gradient tensor $\mathbf{F}$ during growth of an elastic material. A deformation gradient $\mathbf{M}^{e} \mathbf{M}^{g}$ describes the growth relative to the arbitrary, fixed reference configuration $\kappa_{R}(\mathcal{B})$ with residual stress $\stackrel{\circ}{\mathbf{T}}$. An additional elastic deformation gradient due to applied loading (t) is represented by $\mathbf{F}^{l}$.

deposition are described by $\mathbf{M}^{g}$ and that the mass and internal energy density functions are independent of $\mathbf{M}^{g}$. In Section 5, the tensors $\mathbf{M}^{g}$ and $\mathbf{M}^{e}$ will be shown to have a clear physical interpretation in the context of a single increment of growth.

As usual, the velocity $\mathbf{v}$-and the velocity gradient $\mathbf{L}$-are defined as

$$
\mathbf{v}=\frac{\partial \chi(\mathbf{X}, t)}{\partial t}, \quad \mathbf{L}=\frac{\partial \mathbf{v}}{\partial \mathbf{x}}
$$

and the corresponding rate of deformation tensor $\mathbf{D}$-and spin tensor $\mathbf{W}$-is

$$
\mathbf{D}=\frac{1}{2}\left(\mathbf{L}+\mathbf{L}^{T}\right), \quad \mathbf{W}=\frac{1}{2}\left(\mathbf{L}-\mathbf{L}^{T}\right),
$$

respectively. The material time derivative of $\mathbf{F}$-is

$$
\dot{\mathbf{F}}=\mathbf{L F} .
$$

The standard definitions (2.6-2.8) will now be used with (2.5) to derive several important kinematic relations that are needed in Section 4. With (2.5) and (2.8), the velocity gradient may be expressed as

$$
\mathbf{L}=\dot{\mathbf{F}} \mathbf{F}^{-1}=\dot{\mathbf{F}}^{l} \mathbf{F}^{l^{-1}}+\mathbf{F}^{l} \dot{\mathbf{M}}^{e} \mathbf{M}^{e^{-1}} \mathbf{F}^{l^{-1}}+\mathbf{F}^{l} \mathbf{M}^{e} \dot{\mathbf{M}}^{g} \mathbf{M}^{g^{-1}} \mathbf{M}^{e^{-1}} \mathbf{F}^{l^{-1}}
$$


With the definitions

$$
\mathbf{L}^{l}=\dot{\mathbf{F}}^{l} \mathbf{F}^{l^{-1}}, \quad \mathbf{L}^{e}=\dot{\mathbf{M}}^{e} \mathbf{M}^{e^{-1}}, \quad \mathbf{L}^{g}=\dot{\mathbf{M}}^{g} \mathbf{M}^{g^{-1}},
$$

(2.9) becomes ${ }^{1}$

$$
\mathbf{L}=\mathbf{L}^{l}+\mathbf{F}^{l} \mathbf{L}^{e} \mathbf{F}^{l^{-1}}+\mathbf{F}^{l} \mathbf{M}^{e} \mathbf{L}^{g} \mathbf{M}^{e^{-1}} \mathbf{F}^{l^{-1}}
$$

The symmetric and skew parts of $\left(\mathbf{L}^{l}, \mathbf{L}^{e}, \mathbf{L}^{g}\right)$ are defined as in (2.7) and are denoted as $\left(\mathbf{D}^{l}, \mathbf{D}^{e}, \mathbf{D}^{g}\right)$ and $\left(\mathbf{W}^{l}, \mathbf{W}^{e}, \mathbf{W}^{g}\right)$, respectively. Using (2.3), (2.8), (2.10) $)_{3}$, and the standard result that, for any nonsingular tensor $\mathbf{A}$,

we obtain

$$
\frac{\cdot}{\operatorname{det} \mathbf{A}}=(\operatorname{det} \mathbf{A}) \mathbf{A}^{-T} \cdot \dot{\mathbf{A}}
$$

$$
\frac{\dot{J}}{J}=\mathbf{D} \cdot \mathbf{1}, \quad \frac{\frac{\cdot}{\operatorname{det} \mathbf{M}^{g}}}{\operatorname{det} \mathbf{M}^{g}}=\mathbf{D}^{g} \cdot \mathbf{1} .
$$

Let the effective elastic deformation $\mathbf{F}^{*}$ and the effective right Cauchy Green deformation $\mathbf{C}^{*}$ be defined as

$$
\mathbf{F}^{*}=\mathbf{F}^{l} \mathbf{M}^{e}, \quad \mathbf{C}^{*}=\mathbf{F}^{*^{T}} \mathbf{F}^{*} .
$$

Clearly, $\mathbf{C}^{*}$ is properly invariant under superposed rigid-body motions. With $(2.10)_{1,2}$ and (2.14), the material time derivative of $\mathbf{C}^{*}$ becomes

$$
\dot{\mathbf{C}}^{*}=\mathbf{M}^{e^{T}} \mathbf{L}^{e^{T}} \mathbf{F}^{l^{T}} \mathbf{F}^{l} \mathbf{M}^{e}+2 \mathbf{M}^{e^{T}} \mathbf{F}^{l^{T}} \mathbf{D}^{l} \mathbf{F}^{l} \mathbf{M}^{e}+\mathbf{M}^{e^{T}} \mathbf{F}^{l^{T}} \mathbf{F}^{l} \mathbf{L}^{e} \mathbf{M}^{e} .
$$

\section{3.- BALANCE-LAWS-}

In this section, the procedure outlined by Atkin and Craine [24] for obtaining the balance laws for a single constituent in a reacting mixture will be closely followed. However, some interaction terms included in [24] are not necessary here as we are interested in obtaining the balance laws for a single constituent (i.e., the growing elastic material). In addition to the balance laws, a growth continuity equation will be defined. An important assumption in our development is that the material deposited during growth has the same mechanical properties as the original material. This assumption has two implications. First, the mass density, linear momentum, angular momentum, internal energy, and kinetic energy of the deposited material are the same as that of the original material at a point. Second, the mechanical response functions of the deposited material are the same as those of the original material.

We recall that the set of original material points in the reference configuration is dense in the set of all points in the grown configuration. A common approach used in formulating 
the balance laws for a continuum involves the definition of a material region of the body that always contains the same material particles. Since a material region is more difficult to define for a growing material that may be adding or losing material points, it is more straightforward to formulate the balance laws using a region fixed in space. Thus, we consider an arbitrary region $\mathcal{P}$ fixed in $\mathcal{E}^{3}$ and bounded by a closed surface $\partial \mathcal{P}$ with unit outward normal $\mathbf{n}$. It is emphasized that the balance laws are posed for the loaded grown configuration $\kappa(\mathcal{B})$ at time $t$.

\subsection{Balance of Mass}

The integral form of the balance of mass is

$$
\frac{\partial}{\partial t} \int_{\mathcal{P}} \rho d V+\int_{\partial \mathcal{P}} \rho \mathbf{v} \cdot \mathbf{n} d A=\int_{\mathcal{P}} \rho c d V
$$

where the mass density of the deposited material is the same as that of the existing material at a point. The quantity $\rho c$ is the mass addition due to growth and has units of (mass/volumetime). Thus, the mass growth function $c$ represents the amount of mass deposited per unit time per unit current mass. By using (3.1) and following standard procedures [25], we derive the local continuity equation: ${ }^{2}$

$$
\dot{\rho}+\rho \operatorname{div} \mathbf{v}=\rho c .
$$

Recalling $(2.13)_{1}$, (3.2) may be integrated to yield the material form of the continuity equation:

$$
\rho J=\rho_{0} \exp \left[\int_{\tau=t_{0}}^{t} c d \tau\right] .
$$

In [22], only incompressible materials were considered, so the term on the right-hand side of (3.2) was represented by the volume growth function $(\dot{m})$ with units of (mass/volume-time). Thus, (3.3) could be derived using $\dot{m}=\rho c$; the result is

$$
\rho J=\rho_{0} \exp \left[\int_{\tau=t_{0}}^{t} \frac{\dot{m}}{\rho} d \tau\right] .
$$

We prefer to use the mass growth function $c$ and (3.3) because the mass density function $\rho$ does not appear in the integrand as it does in (3.4).

The mass growth function $c$ is not a standard variable in the balance of mass equation, and it requires the introduction of an additional equation. Thus, a growth continuity equation is formulated by assuming that the density of the material only depends on the effective elastic deformation tensor $\mathbf{F}^{l} \mathbf{M}^{e}$. Accordingly, we assume

$$
\rho J^{*}=\rho_{0},
$$

where $J^{*}=\operatorname{det} \mathbf{F}^{l} \operatorname{det} \mathbf{M}^{e}$. The result (3.5) was obtained in [21] for a growing compressible elastic material. Together, (2.5), (3.3), and (3.5) produce the growth continuity equation 


$$
\operatorname{det} \mathbf{M}^{g}=\exp \left[\int_{\tau=t_{0}}^{t} c d \tau\right]
$$

or, equivalently,

$$
\frac{\overline{\operatorname{det} \mathbf{M}^{g}}}{\operatorname{det} \mathbf{M}^{g}}=c
$$

\subsection{Balance of Linear Momentum}

The integral form of the balance of linear momentum is

$$
\frac{\partial}{\partial t} \int_{\mathcal{P}} \rho \mathbf{v} d V+\int_{\partial \mathcal{P}} \rho(\mathbf{v} \cdot \mathbf{n}) d A=\int_{\mathcal{P}} \rho \mathbf{b} d V+\int_{\partial \mathcal{P}} \mathbf{t} d A+\int_{\mathcal{P}} \rho c \mathbf{v} d V,
$$

where $\mathbf{b}$-is the external body force and $\mathbf{t}$-is the traction vector. The last integral represents the linear momentum increase due to mass deposition, where the linear momentum of the deposited material is the same as that of the existing material at a point. With the continuity equation (3.2), we obtain the local form of (3.8): ${ }^{3}$

$$
\operatorname{div} \mathbf{T}+\boldsymbol{\rho} \mathbf{b}=\rho \dot{\mathbf{v}},
$$

where $\mathbf{T}$-is the Cauchy stress tensor. Note that the last integral on the right-hand side of (3.8) does not appear in the local form (3.9), due to the mass growth function that appears on the right-hand side of the continuity equation (3.2).

\subsection{Balance of Angular Momentum}

The integral form of the balance of angular momentum referred to an arbitrary fixed position vector $\mathbf{x}_{0}$ is

$$
\begin{aligned}
& \frac{\partial}{\partial t} \int_{\mathcal{P}} \rho\left(\mathbf{x}-\mathbf{x}_{0}\right) \times \mathbf{v} d V+\int_{\partial \mathcal{P}} \rho\left(\mathbf{x}-\mathbf{x}_{0}\right) \times \mathbf{v}(\mathbf{v} \cdot \mathbf{n}) d A \\
= & \int_{\mathcal{P}}\left(\mathbf{x}-\mathbf{x}_{0}\right) \times \rho \mathbf{b} d V+\int_{\partial \mathcal{P}}\left(\mathbf{x}-\mathbf{x}_{0}\right) \times \mathbf{t} d A \\
+ & \int_{\mathcal{P}} \rho c\left(\mathbf{x}-\mathbf{x}_{0}\right) \times \mathbf{v} d V
\end{aligned}
$$

The last integral represents the angular momentum increase due to mass deposition, where the angular momentum of the deposited material is the same as that of the existing material at a point. ${ }^{4}$ With (3.2) and (3.9), we obtain the local form of (3.10): 


$$
\mathbf{T}=\mathbf{T}^{T}
$$

Note that, as before, the last integral on the right-hand side of (3.10) does not contribute an extra term in the local form (3.11).

\section{4.- WORK-ENERGY-PRINCIPLE-}

There are various ways to derive the general constitutive restriction for Green-elastic materials. Here we follow the approach where the time rate of change of mechanical energy is assumed to equal the total mechanical power (e.g., see Beatty [26]). In this approach, one assumes a priori the existence of a strain energy density (or internal energy density) function $\varepsilon{ }^{5}$ The work-energy principle for the growth of an elastic material can be stated as follows:

The time rate of change of internal and kinetic energy is equal to the work done by body forces and surface tractions, the rate of change of internal and kinetic energy due to mass deposition, and the growth energy supplied.

Thus, we write the integral form of the work-energy principle as

$$
\begin{aligned}
& \frac{\partial}{\partial t} \int_{\mathcal{P}} \rho\left(\varepsilon+\frac{1}{2} \mathbf{v} \cdot \mathbf{v}\right) d V+\int_{\partial \mathcal{P}} \rho\left(\varepsilon+\frac{1}{2} \mathbf{v} \cdot \mathbf{v}\right)(\mathbf{v} \cdot \mathbf{n}) d A \\
= & \int_{\mathcal{P}} \rho \mathbf{b} \cdot \mathbf{v} d V+\int_{\partial \mathcal{P}} \mathbf{t} \cdot \mathbf{v} d A+\int_{\mathcal{P}} \rho c\left(\varepsilon+\frac{1}{2} \mathbf{v} \cdot \mathbf{v}\right) d V+\int_{\mathcal{P}} \rho \beta d V .
\end{aligned}
$$

The third integral on the right-hand side of (4.1) is the rate of change of energy due to mass deposition, where we have assumed that the internal and kinetic energy of the material deposited is the same as that of the existing material at that point. This agrees with our basic assumption that the material deposited is mechanically equivalent to the existing material. In the last integrand on the right-hand side of (4.1), the term $B$ is the rate of growth energy (per unit current mass) that is required in addition to that needed to create material with the same internal and kinetic energy as the existing material. Using the balance equations derived in Section 3, we obtain the local form of (4.1): ${ }^{6}$

$$
\rho \dot{\varepsilon}-\rho \beta=\mathbf{T} \cdot \mathbf{D} .
$$

Recall that $\mathbf{T}$-is symmetric, so with (2.10), (4.2) may be expressed as

$$
\rho \dot{\varepsilon}-\rho ß-\mathbf{T} \cdot\left(\mathbf{L}^{l}+\mathbf{F}^{l} \mathbf{L}^{e} \mathbf{F}^{l^{-1}}+\mathbf{F}^{l} \mathbf{M}^{e} \mathbf{L}^{g} \mathbf{M}^{e^{-1}} \mathbf{F}^{l^{-1}}\right)=0 .
$$

To derive constitutive restrictions from (4.3), it is assumed that the internal energy is a function of the effective elastic deformation and the residual stress, that is, 


$$
\varepsilon=\tilde{\varepsilon}\left(\mathbf{F}^{l} \mathbf{M}^{e}, \stackrel{\circ}{\mathbf{T}}\right)=\left(\mathbf{F}^{*}, \stackrel{\circ}{\mathbf{T}}\right) .
$$

To satisfy invariance under superposed rigid-body motions, (4.4) must be expressible as

$$
\varepsilon=\hat{\varepsilon}\left(\mathbf{C}^{*}, \stackrel{\circ}{\mathbf{T}}\right) .
$$

We emphasize that because the material is elastic, the Cauchy stress is rate independent. By introducing (2.15) and (4.5) into (4.3), using the chain rule and noting that the residual stress field in the configuration $\kappa_{R}(\mathcal{B})$ is fixed, we obtain

$$
\begin{gathered}
\left\{2 \rho \mathbf{F}^{l} \mathbf{M}^{e} \frac{\partial \hat{\varepsilon}}{\partial \mathbf{C}^{*}} \mathbf{M}^{e^{T}} \mathbf{F}^{l^{T}}-\mathbf{T}\right\} \cdot \mathbf{L}^{l} \\
+\left\{2 \rho \mathbf{F}^{l^{T}} \mathbf{F}^{l} \mathbf{M}^{e} \frac{\partial \hat{\varepsilon}}{\partial \mathbf{C}^{*}} \mathbf{M}^{e^{T}}-\mathbf{F}^{l^{T}} \mathbf{T} \mathbf{F}^{l^{-T}}\right\} \cdot \mathbf{L}^{e} \\
-\left\{\mathbf{M}^{e^{T}} \mathbf{F}^{l^{T}} \mathbf{T} \mathbf{F}^{l^{-T}} \mathbf{M}^{e^{-T}}\right\} \cdot \mathbf{L}^{g}-\rho ß=0 .
\end{gathered}
$$

The final constitutive assumption is that both $\dot{\mathbf{M}}^{g}$ and $\beta$ are independent of the time rate of change of the superposed elastic deformation gradient tensor, $\dot{\mathbf{F}}^{l} \cdot{ }^{7}$ Returning to the decomposition $\mathbf{F}=\mathbf{F}^{l}$, note that, at any particular point, the elastic deformation gradient due to loading $\mathbf{F}^{l}$ may be chosen independently from the growth tensor $\mathbf{M}^{g}$ and the elastic accommodation tensor $\mathbf{M}^{e}$ (although $\mathbf{M}^{g}$ and $\mathbf{M}^{e}$ are not independent of each other). Following standard arguments (e.g., see [24]), we obtain ${ }^{8}$

$$
\mathbf{T}=2 \rho \mathbf{F}^{*} \frac{\partial \hat{\varepsilon}}{\partial \mathbf{C}^{*}} \mathbf{F}^{*^{T}} .
$$

Using (4.7), it is trivial to show that the terms in the second set of brackets in (4.6) cancel, leaving

$$
\mathbf{T} \cdot \mathbf{F}^{l} \mathbf{M}^{e} \mathbf{L}^{g} \mathbf{M}^{e^{-1}} \mathbf{F}^{l^{-1}}+\rho ß=0 .
$$

Thus, we have explicitly derived the constitutive restriction (4.7), which states that a growing elastic material behaves as a Green-elastic material, an idea that was assumed in earlier works $[17,22]$. In addition, we have derived the new equation (4.8), which states that the stress power due to growth is balanced by the rate of change of the extra growth energy.

In summary, the governing equations for growth of a compressible elastic material are the balance of mass (3.2), growth continuity (3.6), balance of linear momentum (3.9), balance of angular momentum (3.11), and decomposition equations (2.5). These represent 17 scalar equations for the 32 scalar unknowns obtained from $\rho, \mathbf{v}, \mathbf{c}, \mathbf{T}, \mathbf{M}^{g}$ and $\mathbf{F}^{*}$. Thus, two additional equations are required; one is supplied by the constitutive restriction for the stress (4.7) and provides 6 scalar equations, and a second is required for $\dot{\mathrm{M}}^{g}$ and 
gives 9 scalar equations. ${ }^{9}$ The assumption of the existence of an internal energy function requires the additional constitutive equation (4.5), while the reduced work-energy balance (4.8) determines the additional unknown $B$.

The necessity of including the growth energy supply $\beta$ in the present theory is illustrated by a simple example. Consider a uniform, isotropic growth of a homogeneous material without residual stress for which the components of $\dot{\mathbf{M}}^{g}$ are positive. Furthermore, suppose there exists external loading such that the stress in the material is spherical and compressive. Due to the results obtained for the corresponding boundary-value problem [22], no residual stress develops in the material, and the tensor $\mathbf{M}^{e}=\mathbf{1}$. For this example, it may be easily seen that the stress power due to growth is negative (i.e., $\mathbf{T} \cdot \mathbf{F}^{l} \mathbf{L}^{g} \mathbf{F}^{l^{-1}}<0$ ). Thus, (4.8) reveals that a positive growth energy $\beta$, in addition to the energy of the mass deposited, must be supplied for this growth to be possible.

\section{5.- THE-INCREMENTAL-GROWTH-PROBLEM-}

Growth is a continuous process involving the addition or removal of material points; consequently, the most physically relevant tensorial description of mass deposition is defined in the present configuration of a growing material. Thus, an incremental approach is used to implement the theory; the incremental approach was presented by [22] for incompressible elastic materials. In the incremental approach, the reference configuration $\kappa_{R}(\mathcal{B})$ is chosen to coincide with that obtained by unloading from the configuration $\kappa_{0}(\mathcal{B})$ at time $t_{0}$ (Figure 3), and the governing equations correspond to the static boundary-value problem for an increment of growth from $\kappa_{R}(\mathcal{B})$ to the unloaded grown configuration $\kappa_{g}(\mathcal{B})$. Thus, the only external loads that are considered are those corresponding to the configuration $\kappa_{0}(\mathcal{B})$ and that may be used to determine the growth law relative to the reference configuration $\kappa_{R}(\mathcal{B})$.

In particular, consider an increment of growth with no applied loads such that the deformation gradient tensor of the mapping from $\kappa_{R}(\mathcal{B})$ to $\kappa_{g}(\mathcal{B})$ obeys the decomposition

$$
\mathbf{F}=\mathbf{F}^{e} \mathbf{G} \text {. }
$$

In (5.1), $\mathbf{G}-$ is the incremental growth tensor that describes the amount and orientation of mass deposition, and $\mathbf{F}^{e}$ is the elastic accommodation tensor that ensures compatibility of $\kappa_{g}(\mathcal{B})$. Comparison of (5.1) with (2.5) evaluated at $\mathbf{F}^{l}=\mathbf{1}$ suggests that

$$
\mathbf{M}^{e}(\mathbf{X}, t)=\mathbf{F}^{e}(\mathbf{X}, t), \quad \mathbf{M}^{g}(\mathbf{X}, t)=\mathbf{G}(\mathbf{X}, t)
$$

in the growth boundary-value problem for one increment. Note that an arbitrary, orthogonal tensor $\mathbf{Q}$-may appear in each of (5.2) such that $\mathbf{F}^{e} \mathbf{G}=\mathbf{M}^{e} \mathbf{Q}^{T} \mathbf{Q} \mathbf{M}^{g}$; here we take $\mathbf{Q}=\mathbf{1}$. The physics of the actual growth process suggest that a constitutive equation is needed for the tensorial description of mass deposition as a function of position $\mathbf{X}_{0}$ in the loaded configuration $\kappa_{0}(\mathcal{B})$. This constitutive equation is termed the growth law and will be denoted by $\overline{\mathbf{G}}$. For example, we may assume that growth depends on the stress in $\kappa_{0}(\mathcal{B})$; then $\overline{\mathbf{G}}\left(\mathbf{X}_{0}, t_{0} ; \mathbf{T}_{\kappa_{0}}\right)$. Using the mapping from $\kappa_{0}(\mathcal{B})$ to $\kappa_{R}(\mathcal{B})$, the growth law may be rewritten 


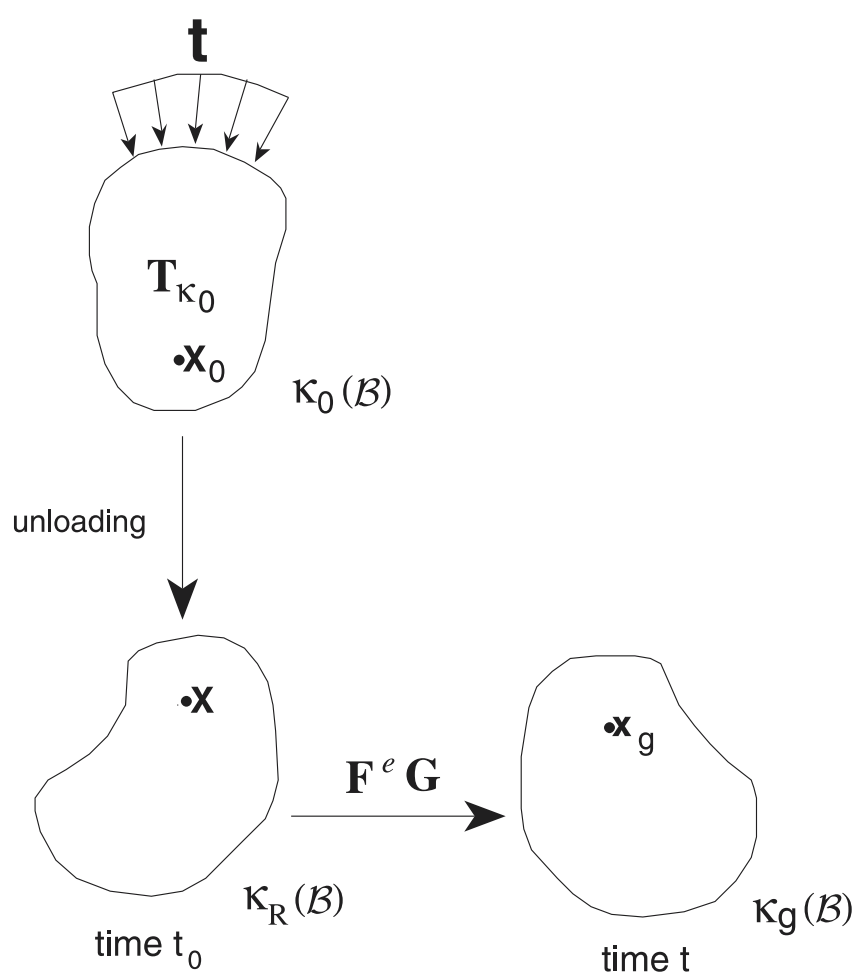

Fig. 3. An increment of growth from the configuration $\kappa_{R}(\mathcal{B})$ at time $t_{0}$ to the configuration $\kappa_{g}(\mathcal{B})$ at time $t$. The configuration $\kappa_{R}(\mathcal{B})$ corresponds to the loaded configuration $\kappa_{0}(\mathcal{B})$ with stress $\mathbf{T}_{\kappa_{0}}$ at time $t_{0}$. The deformation gradient of the mapping from $\kappa_{R}(\mathcal{B})$ to $\kappa_{g}(\mathcal{B})$ is decomposed as $\mathbf{F}^{e} \mathbf{G}$, where $\mathbf{G}$ is the incremental growth tensor and $\mathbf{F}^{e}$ is the elastic accommodation tensor.

as a function of position $\mathbf{X}$ in the unloaded configuration $\kappa_{R}(\mathcal{B})$. Thus, the time rate of change of the incremental growth tensor, $\dot{\mathbf{G}}$, is given by

$$
\dot{\mathbf{G}}=\tilde{\mathbf{G}}\left(\mathbf{X}, t_{0} ; \mathbf{T}_{\kappa_{0}}\right) \text {. }
$$

Since the growth law (5.3) is specified in $\kappa_{R}(\mathcal{B})$, comparison with $(2.10)_{3}$ evaluated at $\mathbf{M}^{g}=\mathbf{1}$ and $(5.2)_{2}$ reveals that

$$
\mathbf{L}^{g}\left(\mathbf{X}, t_{0}\right)=\dot{\mathbf{M}}^{g}\left(\mathbf{X}, t_{0}\right)=\dot{\mathbf{G}}\left(\mathbf{X}, t_{0}\right)
$$

in the growth boundary-value problem for one increment. Using a Taylor series expansion about time $t_{0}$, to first order

$$
\mathbf{G}(\mathbf{X}, t)=\mathbf{1}+\left(t-t_{0}\right) \dot{\mathbf{G}}\left(\mathbf{X}, t_{0}\right) .
$$


With the observations (5.2) and (5.4) and recalling $(2.13)_{2}$, the growth continuity equation (3.7) evaluated in $\kappa_{R}(\mathcal{B})$ may be written in terms of the incremental growth tensor as

$$
c\left(\mathbf{X}, t_{0}\right)=\frac{\cdot}{\operatorname{det} \mathbf{G}\left(\mathbf{X}, t_{0}\right)}=\dot{\mathbf{G}}\left(\mathbf{X}, t_{0}\right) \cdot \mathbf{1} .
$$

When the time increment is sufficiently small, the mass growth function $c$ may be considered as a constant, and (5.6) $)_{1}$ becomes, to first order,

$$
\operatorname{det} \mathbf{G}(\mathbf{X}, t)=\mathbf{1}+\left(t-t_{0}\right) c\left(\mathbf{X}, t_{0}\right) .
$$

In addition to (5.1), (5.5), and (5.7), the governing equations for incremental growth of a compressible elastic material are the balance of mass

$$
\rho J^{e}=\rho_{0},
$$

equilibrium

$$
\operatorname{div} \mathbf{T}=\mathbf{0},
$$

balance of angular momentum

$$
\mathbf{T}=\mathbf{T}^{T},
$$

and stress constitutive equations

$$
\mathbf{T}=2 \rho \mathbf{F}^{e} \frac{\partial \hat{\varepsilon}}{\partial \mathbf{C}^{e}} \mathbf{F}^{e^{T}},
$$

where $J^{e}=\operatorname{det} \mathbf{F}^{e}$. In addition, the constitutive restriction (4.8) evaluated in $\kappa_{R}(\mathcal{B})$ reduces to

$$
\mathbf{T} \cdot \dot{\mathbf{G}}+\rho ß=0,
$$

which reveals that the growth energy supply $\rho ß$ is balanced by the stress power due to growth when evaluated in the present configuration of a growth process.

To formulate a boundary-value problem that includes a superposed elastic deformation, the traction $\mathbf{t}=\mathbf{T n}$ may be prescribed on a subset of the boundary $\partial \mathcal{R}_{t} \subseteq \partial \mathcal{R}$, and the motion $\mathbf{x}$-may be prescribed on a subset of the boundary $\partial \mathcal{R}_{\mathbf{x}} \subseteq \partial \mathcal{R}$, where $\partial \mathcal{R}$ is the boundary of the body $\mathcal{B}$ in the loaded, grown configuration.

\section{6.- EXAMPLES-}

In this section, several examples are presented to illustrate the main features of the theory. In particular, we study growth of a spherical shell using two strain energy functions that have been used to describe biological materials. 


\subsection{Geometry and Boundary Conditions}

We consider the growth of a spherical shell from an unloaded initial configuration that is stress free to a deformed spherical shell in an unloaded final configuration. The inner and outer radii of the spherical shell in the initial configuration are equal to 1 and 2, respectively. Since an incremental solution technique is used, the problem is posed in this section as an incremental growth from an unloaded reference configuration with residual stress to an unloaded grown configuration. To retain spherical symmetry, both the growth and the elastic accommodation tensors must be spherically symmetric and, consequently, depend only on the radial coordinate. Thus, for an increment of growth, the overall motion of the body is

$$
r=\hat{r}(R), \quad \theta=\Theta, \quad \phi=\Phi,
$$

where $(r, \theta, \phi)$ and $(R, \Theta, \Phi)$ are the spherical coordinates in the grown and reference configurations, respectively. The basis vectors in the reference configuration are $\left(\mathbf{e}_{R}, \mathbf{e}_{\Theta}, \mathbf{e}_{\Phi}\right)$. The inner and outer boundaries of the shell in the reference configuration are denoted $R=R_{i}$ and $R=R_{o}$, respectively. The traction boundary condition $\mathbf{t}=\mathbf{0}$ at the inner and outer boundaries results in the nontrivial equations

$$
\begin{aligned}
& T_{r r}\left(\hat{r}\left(R_{i}, t\right), t\right)=0, \\
& T_{r r}\left(\hat{r}\left(R_{0}, t\right), t\right)=0,
\end{aligned}
$$

where $T_{r r}$ is the normal stress component in the radial direction.

\subsection{Growth Specification}

The incremental approach described in Section 5 is used to solve the growth boundary-value problem. We consider isotropic growth and two special cases of anisotropic growth: radial and circumferential. Isotropic, radial, and circumferential growth tensors are represented as in [22] by

$$
\begin{aligned}
\mathbf{G}_{\text {iso }} & =\lambda^{g} \mathbf{l}, \\
\mathbf{G}_{\mathrm{rad}} & =\lambda_{R}^{g} \mathbf{e}_{R} \otimes \mathbf{e}_{R}+\mathbf{e}_{\Theta} \otimes \mathbf{e}_{\Theta}+\mathbf{e}_{\Phi} \otimes \mathbf{e}_{\Phi}, \\
\mathbf{G}_{\mathrm{cir}} & =\mathbf{e}_{R} \otimes \mathbf{e}_{R}+\lambda_{\Theta}^{g} \mathbf{e}_{\Theta} \otimes \mathbf{e}_{\Theta}+\lambda_{\Theta}^{g} \mathbf{e}_{\Phi} \otimes \mathbf{e}_{\Phi},
\end{aligned}
$$

respectively. An isotropic growth tensor may be visualized as an infinitesimal sphere of material growing into a larger or smaller sphere, while an anisotropic growth tensor may be visualized as an infinitesimal sphere of material growing into an ellipsoid. In particular, the radial growth tensor $\mathbf{G}_{\mathrm{rad}}$ represents mass deposition only in the radial direction, while 
the circumferential growth tensor $\mathbf{G}_{\text {cir }}$ represents mass deposition only in the circumferential direction. For each boundary-value problem, a total mass increase of $1 \%$ of the current mass is specified in each of 50 increments, resulting in a total mass increase of $64.9 \%$. To explore the effects of nonhomogeneous growth, two cases are considered.

\subsubsection{Case 1: Uniform Growth}

Here the mass growth function $c$ is uniform through the thickness of the shell. We specify $c=0.01$ for each increment and, from (5.7) and (6.3) and $t-t_{0}=1$, the growth stretches for isotropic, radial, and circumferential growth are calculated as

$$
\begin{aligned}
& \lambda^{g}=(1+c)^{1 / 3}, \\
& \lambda_{R}^{g}=1+c, \\
& \lambda_{\Theta}^{g}=(1+c)^{1 / 2},
\end{aligned}
$$

respectively.

\subsubsection{Case 2: Nonuniform Growth}

Here the mass growth function $c(R)$ is a linear function of the radial position in the reference configuration, from $c=0$ at the inner radius $R_{i}$ to $c=c_{\max }$ at the outer radius $R_{o}$. In particular, $c_{\max }$ is calculated to achieve a total mass increase of $1 \%$ of the current mass in each increment. Once $c(R)$ is specified, the growth stretches are calculated as in (6.4) as a function of radial position.

\subsection{Constitutive Equations}

Two constitutive equations with exponential strain energy functions were chosen that exhibit the strong tension-compression asymmetry observed for cartilaginous tissues. In these tissues, the tensile response is attributed to the presence of collagen fibers that offer little resistance in compression but allow the material to support high tensile forces. These tissues typically have a tangent modulus in uniaxial tension experiments that is one to two orders of magnitude higher than the compressive stiffness. The present study uses two strain energy functions obtained by neglecting the anisotropic terms from the following models: the transversely isotropic model proposed for articular cartilage by Almeida and Spilker [27] (referred to as the AS model) and the orthotropic model proposed for the annulus fibrosus of the intervertebral disk by Wagner et al. [28] and Klisch and Lotz [29] (referred to as the WKL model). Upon neglecting the anisotropic terms, the AS strain energy function becomes

$$
W=\rho_{0} \varepsilon=\frac{\alpha_{0}}{I_{3}^{\beta}} \exp \left[\alpha_{1}\left(I_{1}-3\right)+\alpha_{2}\left(I_{2}-3\right)+\alpha_{3}\left(I_{1}-3\right)^{2}\right],
$$


where $I_{1}, I_{2}$, and $I_{3}$ are the invariants of the right Cauchy-Green strain tensor, and $\left(\alpha_{0}, \alpha_{1}, \alpha_{2}, \alpha_{3}, \beta\right)$ are material constants equal to $(0.08,7.733,-3.562,1.905,0.6)$. Similarly, the WKL strain energy function becomes

$$
W=\rho_{0} \varepsilon=\alpha_{0}\left\{\exp \left[\alpha_{1}\left(I_{1}-3\right)\right]+\exp \left[\alpha_{2}\left(I_{2}-3\right)\right]+\exp \left[\alpha_{3}\left(I_{3}-1\right)\right]\right\}
$$

where $\left(\alpha_{0}, \alpha_{1}, \alpha_{2}, \alpha_{3}\right)$ are material constants equal to $(0.00138,1.27,2.63,-6.53)$. For the deformations corresponding to the boundary-value problems studied in this paper, it may be shown that the AS material is more than an order of magnitude "stiffer" than the WKL material. Also, the WKL material behaves in a nearly incompressible fashion, with an infinitesimal Poisson's ratio of 0.48 .

\subsection{Solution Procedure}

For each material, a total of six problems were solved. Both uniform and linear mass growth functions were specified with isotropic, radial, and circumferential growth tensors. In all examples, the mass increase of $1 \%$ in each increment and the overall mass increase of $64.9 \%$ were the same. A finite element program for growth elasticity developed by [23] was used to solve the equilibrium equations for the growth boundary-value problem with zero external loads. The output values that are calculated are the Cauchy stress components, the displacements of the material points, and the normalized density function in the final configuration.

\section{7.- RESULTS-AND-DISCUSSION-}

The mechanical response to growth for the two materials is different in a number of ways. The shapes of the residual stress curves for both materials are qualitatively similar for all growth cases; the residual stress plots for linear circumferential growth are shown in Figure 4. Figure 4 reveals that the stress gradients near the inner radius are greater for the WKL material than the AS material, which will be further discussed below. Another difference between the two materials is that the shapes of the radial displacement curves are qualitatively different; for example, for linear circumferential growth, the radial displacement for the AS material was monotonically increasing through most of the shell thickness, while for the WKL material, it was not (Figure 5). Since the response of the WKL material is very similar to that reported for incompressible materials studied earlier [22], only the AS material response is shown below to explore the effects related to nonuniform and anisotropic growth.

The mechanical response to growth varies depending on whether uniform (i.e., homogeneous) or linear (i.e., nonhomogeneous) growth functions are specified. The stress plots for uniform versus linear growth for the AS material are shown for radial growth (Figure 6) and circumferential growth (Figure 7). For radial growth, the uniform case results in higher peak stresses, higher circumferential stresses, and higher stress gradients near the inner radius. 
Table 1. Percent volume change for the AS and the WKL materials for all growth cases.

\begin{tabular}{lccccccc}
\hline & \multicolumn{3}{c}{ Uniform } & & \multicolumn{3}{c}{ Nonuniform } \\
\cline { 2 - 4 } Material & Isotropic & Radial & Circumferential & & Isotropic & Radial & Circumferential \\
\hline AS & 64.9 & 58.6 & 63.4 & & 60.9 & 59.0 & 51.0 \\
WKL & 64.9 & 61.4 & 64.2 & & 63.3 & 62.3 & 59.7 \\
\hline
\end{tabular}

NOTE: The total mass increase is $64.9 \%$, which equals the value of the percent volume change for uniform isotropic growth.

Table 2. Normalized total strain energy for the AS and the WKL materials for all growth cases.

\begin{tabular}{lccccccc}
\hline & \multicolumn{3}{c}{ Uniform } & & \multicolumn{3}{c}{ Nonuniform } \\
\cline { 2 - 4 } Material & Isotropic & Radial & Circumferential & & Isotropic & Radial & Circumferential \\
\hline AS & 0.0 & 7.11 & 1.0 & & 3.17 & 5.16 & 13.48 \\
WKL & 0.0 & 7.93 & 1.0 & & 3.43 & 5.45 & 15.80 \\
\hline
\end{tabular}

NOTE: The AS (WKL) values are normalized by the total strain energy calculated for the AS (WKL) material in uniform circumferential growth. The strain energy is zero for uniform isotropic growth.

In contrast, for circumferential growth, the linear case results in higher peak stresses and higher stress gradients near the inner radius. In addition, uniform radial growth results in more overall compression than uniform circumferential growth, whereas linear circumferential growth results in more overall compression than linear radial growth (Table 1). Finally, uniform isotropic growth is a compatible deformation that leads to no elastic accommodation so that no residual stresses are developed, whereas linear isotropic growth results in residual stresses (Figure 8).

The mechanical response to growth varies depending on the anisotropy, or direction, of the growth tensor (i.e., uniform, radial, or circumferential). As mentioned above, uniform isotropic growth results in zero residual stresses. Although the radial and circumferential stresses for the uniform anisotropic cases are similar in shape, the stresses for the uniform radial case are opposite in sign to those for the uniform circumferential case (Figures 6 and 7). This latter phenomenon is also observed for the linear radial and circumferential growth cases. In addition, the radial displacement and normalized density curves are substantially different due to growth orientation for both the uniform cases (Figure 9) and the linear cases (Figure 10). The overall compression for linear circumferential growth was substantially greater than the other cases (Table 1).

The AS material behaves differently in a number of ways from both the WKL material and the incompressible material reported in [22]. For uniform radial and circumferential growth, the radial displacement curve for the AS material is nearly linear and monotonically increasing through the shell thickness (Figure 9). In contrast, for radial and circumferential growth of the WKL and the incompressible materials, the radial displacements first decrease and then increase through the shell thickness (e.g., see Figure 5). In addition, the stress gradients near the inner radius are less for the AS material than for the WKL and incompressible materials for all growth cases (e.g., see Figure 4). Also, the density reaches higher maximum and minimum values near the inner and outer radii for the AS material due to its greater compressibility (e.g., see Figure 5). 


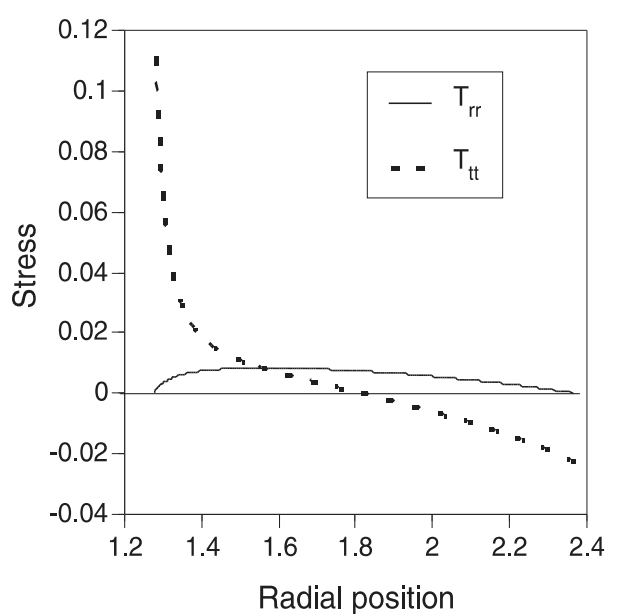

WKL material

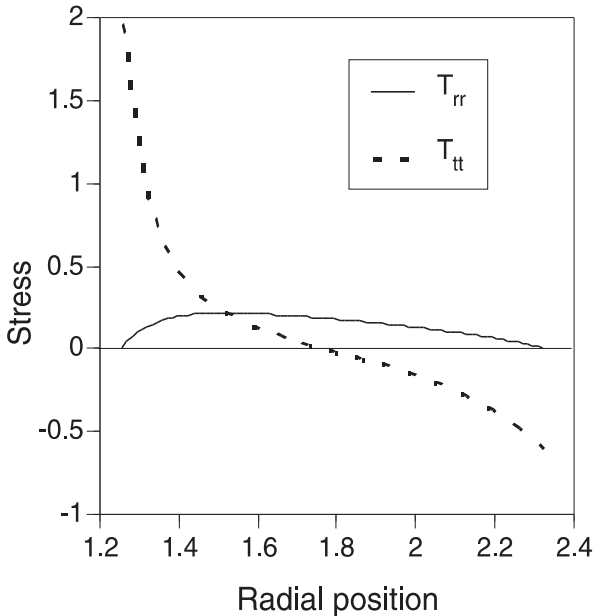

AS material

Fig. 4. Residual stress plots for linear circumferential growth for the WKL (left) and the AS (right) materials. The shapes of both the radial $\left(T_{r r}\right)$ and circumferential $\left(T_{t t}\right)$ stress curves are similar for the two materials. The stress magnitudes for the AS material are an order of magnitude higher than those for the WKL material.
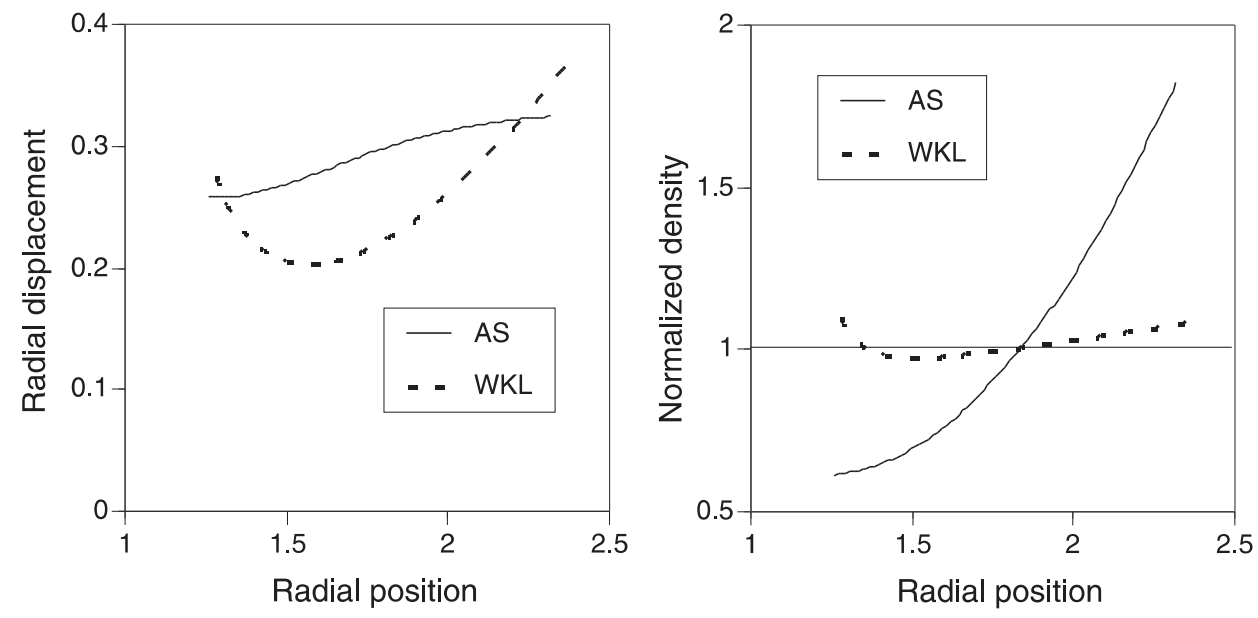

Fig. 5. Radial displacement and normalized density plots for linear circumferential growth for the WKL and the AS materials. The radial displacement for the AS material is monotonically increasing with increasing radius through nearly the whole thickness. The normalized density plots reveal that the AS material is more compressible than the WKL material. 


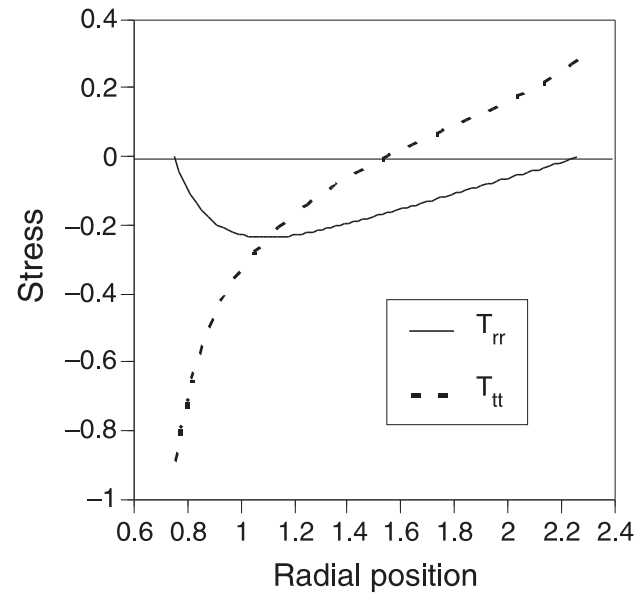

Uniform radial

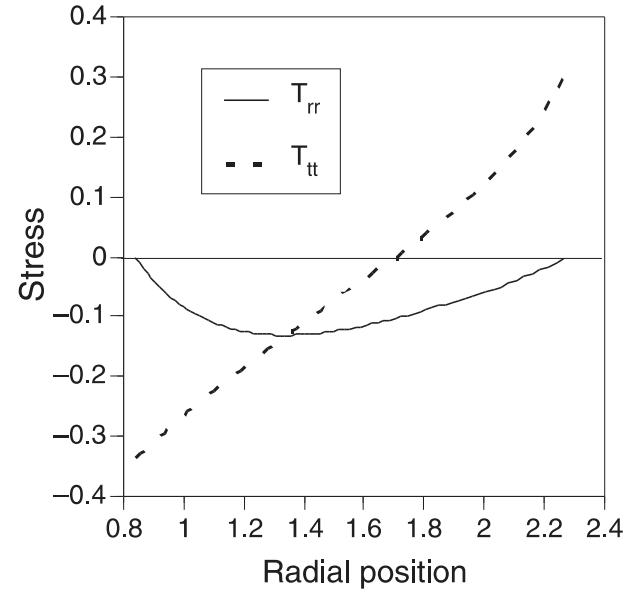

Linear radial

Fig. 6. Stress plots for uniform radial (left) and linear radial (right) growth for the AS material. The uniform case results in higher peak radial stresses, higher circumferential stresses near the inner radius, and higher stress gradients near the inner radius.

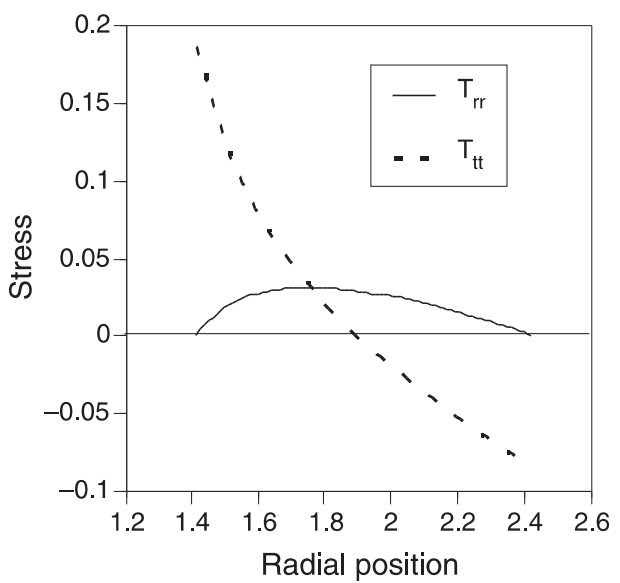

Uniform circumferential

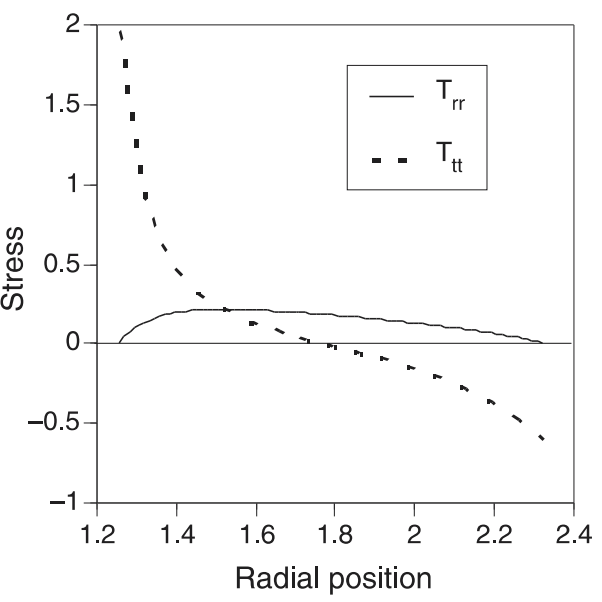

Linear circumferential

Fig. 7. Stress plots for uniform circumferential (left) and linear circumferential (right) growth for the AS material. The linear case results in higher peak stresses and higher stress gradients near the inner radius. 


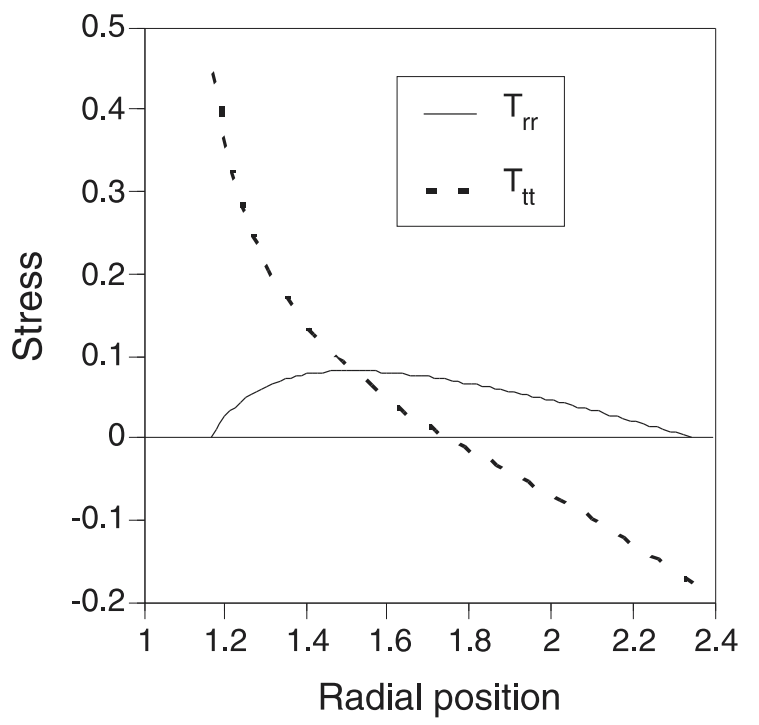

Fig. 8. Stress plot for linear isotropic growth for the AS material (uniform isotropic growth results in zero stresses).
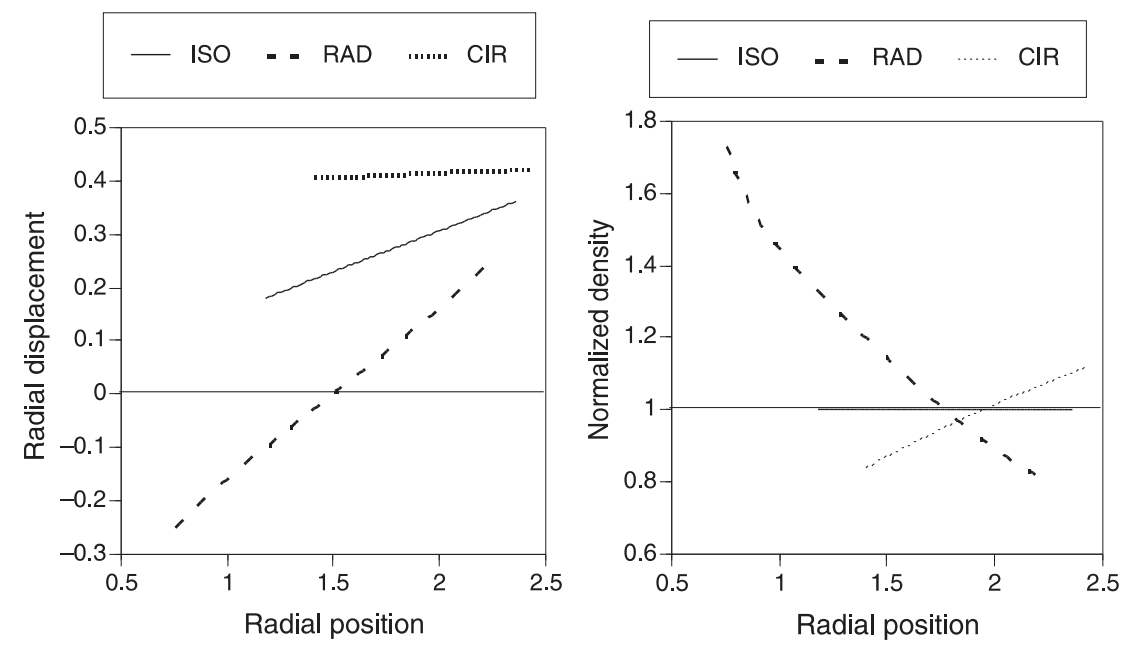

Fig. 9. Radial displacement and normalized density plots for uniform isotropic (ISO), radial (RAD), and circumferential (CIR) growth for the AS material. 

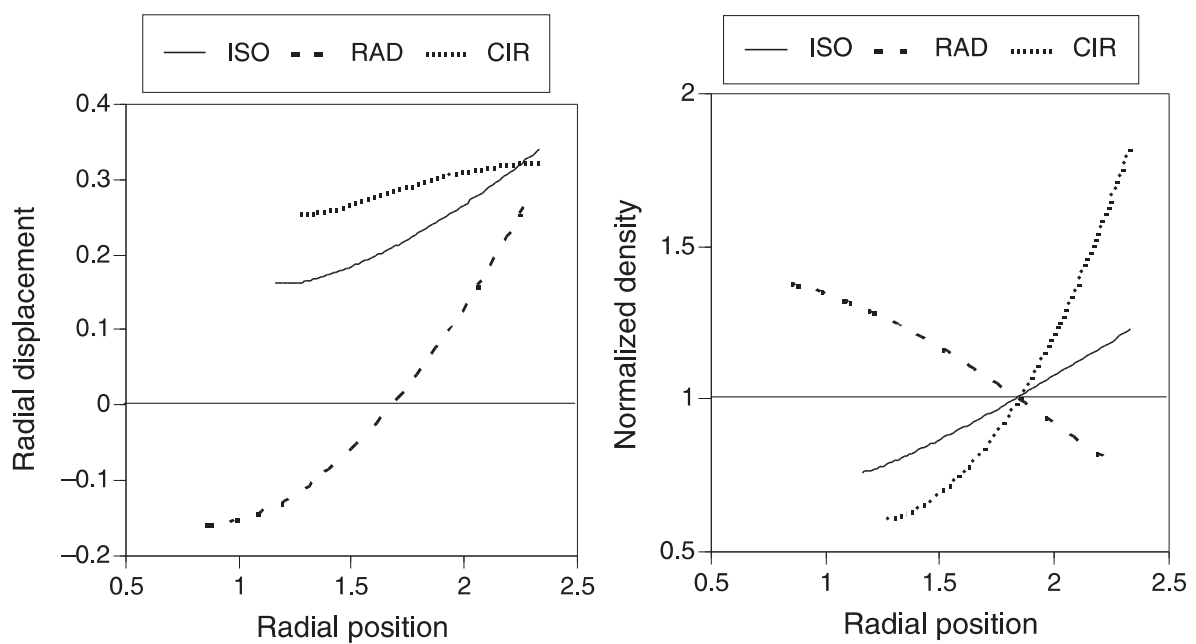

Fig. 10. Radial displacement and normalized density plots for linear isotropic (ISO), radial (RAD), and circumferential (CIR) growth for the AS material.

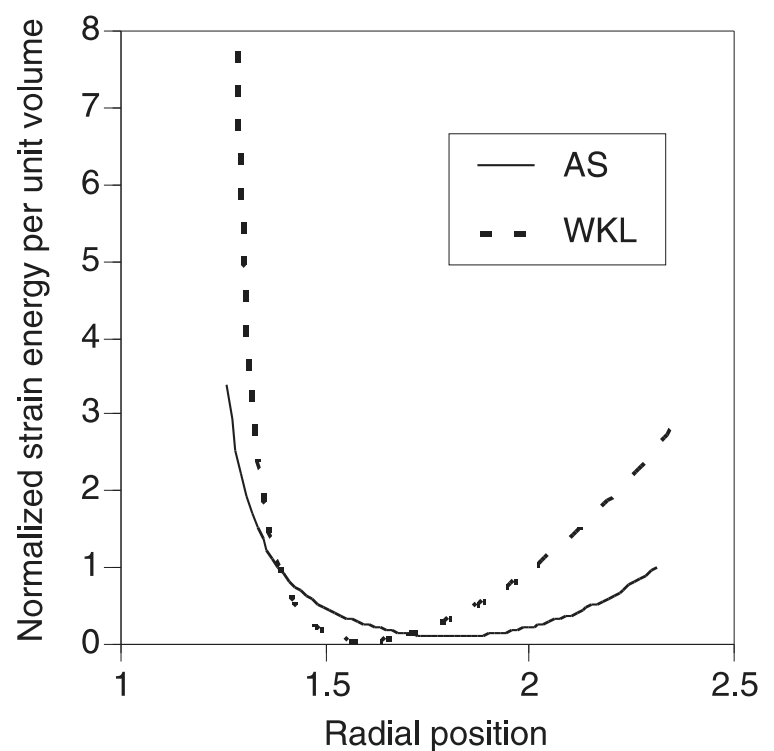

Fig. 11. Normalized strain energy per unit volume plotted versus radial position for the linear circumferential growth for the AS and WKL materials. The strain energy per unit volume was normalized by the material constants $\alpha_{0}$ appearing in the strain energy functions (7.17.2) for the two materials. The strain energy gradient is lower for the AS material. 
To further explore the results that the more compressible AS material achieves lower stress gradients and more linear and monotonic displacement curves than the nearly incompressible WKL material, we considered the stability of an elastic material to perturbations of the boundary conditions in an equilibrium state. Then, it is trivial to show from standard theories on infinitesimal stability (e.g., see [25]) that a stable equilibrium state for a grown elastic material with a convex strain energy function with zero surface tractions and zero body forces is accompanied by a local minima in the total strain energy. ${ }^{10}$ Thus, we calculated the strain energy per unit volume from (6.5) to (6.6), normalized by the material constants $\alpha_{0}$ for the linear circumferential growth case for both materials (Figure 11). It is evident that the gradient in strain energy per unit volume is greatly decreased in the AS material. In addition, for all growth cases, we integrated the strain energy in the grown configuration for the AS and WKL materials to calculate the total strain energy and normalized these values by that achieved in the uniform circumferential case for each material. The results presented in Table 2 show that for each nontrivial growth case, the normalized strain energy was lower for the AS material than for the nearly incompressible WKL material.

To more accurately address the effect of material compressibility on the total strain energy in the grown configuration, it is useful to consider the growth of a spherical shell of a harmonic elastic material for which the compressibility may be changed while holding the shear modulus constant. We studied uniform radial and uniform circumferential growth for the harmonic material with infinitesimal Poisson's ratios of 0.25 and 0.49 , the latter of which corresponds to a nearly incompressible material. As compared to the nearly incompressible harmonic material, the total strain energy in the grown configuration using a Poisson's ratio of 0.25 was $40.1 \%$ and $96.9 \%$ lower for the uniform radial and uniform circumferential growth cases, respectively. Therefore, in these examples, the harmonic material with greater compressibility experienced much lower strain energy states in the grown configuration than the nearly incompressible material. Thus, the differences observed for the displacement and stress profiles for the compressible materials studied in this paper may be coupled and possibly related to achieving a local minima in total strain energy in the grown configuration.

\section{8.- CONCLUSION-}

We have presented a general theory of volumetric growth for compressible elastic materials. In particular, a complete set of governing equations was derived in the present configuration of an elastic material undergoing a continuous growth process. To fully develop the kinematics of growth in the present configuration, we introduced an arbitrary fixed reference configuration and a superposed elastic deformation. Two constitutive restrictions were derived from a workenergy principle. First, we showed that a growing elastic material behaves as a Green-elastic material, an idea that was assumed in earlier works. Second, we obtained an expression that relates the stress power due to growth to the rate of change of energy due to growth. The governing equations for the incremental growth boundary-value problem were obtained from the more general theory, and the main features of the theory were illustrated with specific examples. 
Acknowledgments. The authors are grateful for support from the U.C. President's Office Postdoctoral Fellowship Program (SMK) and grant CMS 9634903 from NSF (AH).

\section{NOTES-}

1. Although the tensors appearing in various equations in this paper are usually defined on different domains, it is clear from (2.1), (2.4), and the comments following (2.4) that they may be defined on the same domain. For example, using (2.4), all quantities appearing in (2.11) may be defined as functions of position $\mathbf{X}$-in the configuration $\kappa_{R}(\mathcal{B})$ and time $t$.

2. The balance of mass (3.2) takes the same form as equation (2.13) in [24] with $m_{\alpha}$ replaced by $\rho c$ and equation (10) in [13] with $c$ replaced by $\rho c$ (quantities in italics in the endnotes refer to those appearing in the noted equations of [24] and [13]).

3. In the balance of linear momentum (3.8), we have neglected the diffusive force arising from interaction with other constituents represented by $p^{\alpha}$ in equation (2.16) of [24] and by $p$ in equation (13) of [13]. Also, we assume that the momentum supply due to growth can be modeled by the velocity $\mathrm{v}$ as in the last integral of (3.8) as opposed to the more general formulation in which $\mathrm{v}$ is replaced by the vector $\mathbf{J}^{\alpha}$ in [24]. This assumption is in agreement with equation (13) of [13]. Thus, the local form (3.9) agrees with equation (2.20) of [24] with $p^{\alpha}=\mathbf{0}$ and $\mathbf{J}^{\alpha}=\mathbf{v}$ and agrees with equation (16) of [13] with $p=\mathbf{0}$.

4. In the balance of angular momentum (3.10), we have assumed $p^{\alpha}=\mathbf{0}, J^{\alpha}=\mathbf{v}$ in equation (2.26) of [24]. Also, we have neglected to include the angular momentum supply due to interaction with other constituents represented by $\boldsymbol{\lambda}^{\alpha}$ in [24]; as a result, our Cauchy stress tensor is symmetric, whereas that in [24] is not. A balance of angular momentum is not explicitly derived in [13].

5. A more primitive approach is to assume that the work done by surface tractions and body forces for an elastic material vanishes for any closed, homogeneous, and smooth cycle through strain space (e.g., see Truesdell and Noll [30]). In this approach, one may then prove the existence of a scalar function, which is related to the stress tensor by the familiar constitutive restriction of Green elasticity. It is clear that this approach is not extendable to growth elasticity.

6. This equation corresponds to the constituent balance of energy (equation (2.33)), presented by [24] with $p^{\alpha}=\mathbf{0}, J^{\alpha}=\mathbf{v}, G^{\alpha}=\varepsilon^{\alpha}+(1 / 2) \mathbf{v}^{\alpha} \cdot \mathbf{v}^{\alpha}$, neglecting the heating terms and replacing the energy increase due to interactions with other constituents with $B$. Furthermore, the balance of energy (equation (17)) in [13] reduces to (4.2) by neglecting the heating terms and setting $\bar{h}$ equal to $\rho ß$.

7. This assumption is a limitation of the theory in the present paper as it excludes the possibility of a dependence on strain rate; see Cowin [31] for a detailed discussion of this point.

8. Since $\dot{\mathbf{F}}^{l}$ may be chosen independently from $\mathbf{F}^{l}$, the relations $\mathbf{L}^{l}=\dot{\mathbf{F}}^{l} \mathbf{F}^{l^{-1}}, \mathbf{L}^{g}=\dot{\mathbf{M}}^{g} \mathbf{M}^{g^{-1}}$, and the assumption that $\dot{\mathbf{M}}^{g}$ does not depend on $\dot{\mathbf{F}}^{l}$ reveal that $\mathbf{L}^{l}$ may be chosen independently from both $\mathbf{L}^{g}$ and $\mathbf{L}^{e}$ (although, again, it is clear that $\mathbf{L}^{g}$ and $\mathbf{L}^{e}$ are not independent of each other). Since the terms in the first set of brackets in (4.6) do not depend on $\mathbf{L}^{l}$, (4.7) follows.

9. The constitutive equation for $\dot{\mathbf{M}}^{g}$ is related to, but not the same as, the standard growth law defined on a current configuration.

10. To be more precise, let $W_{\mathcal{R}}=\int_{\mathcal{R}} W\left(\mathbf{F}^{e}\right) d V$ be the total strain energy of the body $\mathcal{B}$ when $\mathbf{F}^{e}$ corresponds to a stable equilibrium state. Consider an infinitesimal change in the displacement boundary condition on $\partial \mathcal{R}$, corresponding to an effective elastic deformation $\mathbf{F}^{e \prime} \neq \mathbf{F}^{e}$ with $W_{\mathcal{R}}^{\prime}=$ $\int_{\mathcal{R}} W\left(\mathbf{F}^{e \prime}\right) d V$. Then we say that $W_{\mathcal{R}}$ achieves a local minima if $W_{\mathcal{R}} \leq W_{\mathcal{R}}^{\prime}$. 


\section{REFERENCES-}

[1] Pauwels, F: Biomechanics of the Normal and Diseased Hip, Springer-Verlag, New York, 1976.

[2] Ogden, J. A.: Skeletal growth mechanism injury patterns. Behavior of the Growth Plate, ed. H. K. Uhthoff and J. J. Wiley, Raven Press, New York, 1988.

[3] Brickley-Parson, D. and Glimcher, J.: Is the chemistry of collagen in intervertebral discs an expression of Wolff's law? A study of the human lumbar spine. Spine, 9, 148-163 (1984).

[4] Sandy, J. D., Adams, M. E., Billingham, M. E., Plaas, A., and Muir, H.: In vivo and in vitro stimulation of chondrocyte biosynthetic activity in early experimental osteoarthritis. Arthritis and Rheumatism, 27, 388-397 (1984).

[5] Kiviranta, I., Tammi, M., Jurvelin, J., Säämänen, A. M., and Helminen, H. J.: Moderate running exercise augments glycosaminoglycans and thickness of articular cartilage in the knee joint of young beagle dogs. Journal of Orthopaedic Research, 6, 188-195 (1988).

[6] Kiviranta, I., Tammi, M., Jurvelin, J., Arokoski, J., Säämänen, A. M., and Helminen, H. J.: Articular cartilage thickness and glycosaminoglycan distribution in the young canine knee joint after remobilization of the immobilized limb. Journal of Orthopaedic Research, 12, 161-167 (1994).

[7] Palmoski, M., Perricone, E., and Brandt, K. D.: Development and reversal of a proteoglycan aggregation defect in normal canine knee cartilage after immobilization. Arthritis and Rheumatism, 22, 508-517 (1979).

[8] Hall, A. C., Urban, J. P., and Gehl, K. A.: The effects of hydrostatic pressure on matrix synthesis in articular cartilage. Journal of Orthopaedic Research, 9, 1-10 (1991).

[9] Sah, R. L., Kim, Y. J., Doong, J. Y., Grodzinsky, A. J., Plaas, A. H., and Sandy, J. D.: Biosynthetic response of cartilage explants to dynamic compression. Journal of Orthopaedic Research, 7, 619-636 (1989).

[10] Ateshian, G. A., Warden, W. H., Kim, J. J., Grelsamer, R. P., and Mow, V. C.: Finite deformation biphasic material properties of bovine articular cartilage from confined compression experiments. Journal of Biomechanics, 30, 11571164 (1997).

[11] Klisch, S. M. and Lotz, J. C.: A special theory of biphasic mixtures and experimental results for human annulus fibrosus tested in confined compression. Journal of Biomechanical Engineering, 122, 180-188 (2000).

[12] Hsu, F: The influences of mechanical loads on the form of a growing elastic body. Journal of Biomechanics, 1, 303-311 (1968).

[13] Cowin, S. C. and Hegedus, D. M.: Bone remodeling I: A theory of adaptive elasticity. Journal of Elasticity, 6, 313-325 (1976).

[14] Skalak, R., Dasgupta, G., Moss, M., Otten, E., Dullemeijer, P., and Vilmann, H.: Analytical description of growth. Journal of Theoretical Biology, 94, 555-577 (1982).

[15] Skalak, R., Zargaryan, S., Jain, R. K., Netti, P. A., and Hoger, A.: Compatibility and the genesis of residual stress by volumetric growth. Journal of Mathematical Biology, 34, 889-914 (1996).

[16] Skalak, R., Farrow, D. A., and Hoger, A.: Kinematics of surface growth. Journal of Mathematical Biology, 35, 869-907 (1997).

[17] Rodriguez, E. K., Hoger, A., and McCulloch, A. D.: Stress-dependent finite growth in soft elastic tissues. Journal of Biomechanics, 27, 455-467 (1994).

[18] Lin, I. E. and Taber, L.: A model for stress-induced growth in the developing heart. Journal of Biomechanical Engineering, 117, 343-349 (1995).

[19] Taber, L. A. and Eggers, D. W.: Theoretical study of stress-modulated growth in the aorta. Journal of Theoretical Biology, 180, 343-357 (1996).

[20] Taber, L.: A model for aortic growth based on fluid shear and fiber stresses. Journal of Biomechanical Engineering, 120, 348-354 (1998).

[21] Chen, Y. and Hoger, A.: Constitutive function of elastic materials in finite growth and deformation. Journal of Elasticity, 59, 175-193 (2000).

[22] Hoger, A.: Incremental theory of growth for soft biological tissues. Manuscript submitted to the Journal of Elasticity.

[23] Van Dyke, T. J. and Hoger, A.: Should the growth law be defined on the current or the initial configuration? Manuscript submitted to the Journal of Theoretical Biology.

[24] Atkin, R. J. and Craine, R. E.: Continuum theories of mixtures: Basic theory and historical development. Quarterly Journal of Mechanics and Applied Mathematics, 29, 209-244 (1976).

[25] Ogden, R. W.: Non-Linear Elastic Deformations, Dover, New York, 1984.

[26] Beatty, M. F.: Introduction to nonlinear elasticity. Nonlinear Effects in Fluids and Solids, ed. M. M. Carroll and M. A. Hayes, Plenum, New York, 1996.

[27] Almeida, E. S. and Spilker, R. L.: Finite element formulations for hyperelastic transversely isotropic biphasic soft tissues. Computer Methods and Applications in Mechanical Engineering, 151, 513-538 (1998). 
[28] Wagner, D. R., Klisch, S. M., and Lotz, J. C.: A constitutive formulation which simultaneously predicts the response of the annulus fibrosus to seven distinct deformations. BED-Vol. 42, Proceedings of the 1999 Summer Bioengineering Conference, ASME, 363 (1999).

[29] Klisch, S. M. and Lotz, J. C.: Application of a fiber-reinforced continuum theory to multiple deformations of the annulus fibrosus. Journal of Biomechanics, 32, 1027-1036 (1999).

[30] Truesdell, C. and Noll, W.: The non-linear field theories of mechanics. Handbuch der Physik III/3, ed. S. Flügge, Springer-Verlag, Berlin, 1965.

[31] Cowin, S. C.: Strain or deformation rate dependent finite growth in soft tissues. Journal of Biomechanics, 29, 647649 (1996). 